\title{
Conocimientos sobre cáncer de mama y cáncer de cuello uterino en mujeres de Quito: avances de investigación. (1) (9)( ()
}

\section{Knowledge about breast cancer and cervical cancer in women of Quito: advances in research.}

Patricia Hidalgo. ${ }^{1}$, Eduardo Toledo. ${ }^{2}$ \& Juan Carlos García. ${ }^{3}$

\begin{abstract}
.
DOI: https://doi.org/10.33262/cienciadigital.v2i3.141

The rates of affectation due to breast and cervical cancer in Ecuador are among the first causes of death among women. In this regard, different studies have highlighted the importance of generating contributions on these issues. Objective. This article presents the advances of this research that aimed to identify knowledge about breast and cervical cancer, prioritizing aspects related to its causes and methods of prevention in women students and administrative belonging to a higher education institution and women with residence in two sectors of the north and south of the city of quito. Method. A quantitative approach study with an exploratory descriptive scope was used, through the extension of surveys to 384 women from the groups mentioned above. Results. It was identified that the levels of knowledge about causes and prevention of breast cancer are deeper than those of the cervix; in addition, from the perception of the informants, the factors that most influence the risk of contracting this type of cancer are a family history rather than food factors or drug use. A relevant fact is that the best communication strategy, which could strengthen your knowledge is direct contact, through training and brigades in daily places accompanied by media. Conclusions. To conclude, it is emphasized that communication plays an important role when constructing prevention strategies, through the adequate management of spaces and messages that are close to the informants.
\end{abstract}

\footnotetext{
${ }^{1}$ Universidad Internacional del Ecuador, Quito, Ecuador, patrihidal@yahoo.com

${ }^{2}$ Universidad de Las Américas, Quito, Ecuador, alfonso.toledo@udla.edu.ec

${ }^{3}$ Universidad de Las Américas, Quito, Ecuador, jcgarcia@udla.edu.ec
} 
Keywords: Knowledge, Breast Cancer, Cervical Cancer, Communication.

\section{Resumen.}

Las tasas de afectación por cáncer de mama y cuello uterino en ecuador constan dentro de las primeras causas de mortandad de mujeres. En tal sentido, diferentes estudios han marcado la relevancia de generar aportes sobre estos temas. Objetivo. En este artículo se presentan los avances de esta investigación que tuvo como fin identificar los conocimientos sobre cáncer de mama y cuello uterino, priorizando aspectos relacionados a sus causas y métodos de prevención en mujeres estudiantes y administrativas pertenecientes a una institución de educación superior y a mujeres con residencia en dos sectores del norte y sur de la ciudad de quito. Método. Se recurrió a un estudio de enfoque cuantitativo de alcance descriptivo exploratorio, mediante la ampliación de encuestas a 384 mujeres de los grupos mencionados anteriormente. Resultados. Se identificó que los niveles de conocimiento sobre causas y prevención de cáncer de mama son más profundos que los de cuello uterino; además, desde la percepción de las informantes, los factores que más influyen en el riesgo de contraer este tipo de cáncer son antecedentes familiares antes que factores alimenticios o el consumo de drogas. Un dato relevante es que la mejor estrategia de comunicación, que podría fortalecer su conocimiento es el contacto directo, mediante capacitaciones y brigadas en lugares cotidianos acompañados de diferentes medios. Conclusiones. Para concluir se resalta que la comunicación juega un papel relevante al momento de construir estrategias de prevención, mediante la adecuada gestión de espacios y mensajes que sean cercanos a las informantes.

Palabras Claves: Conocimiento, Cáncer de Mama, Cáncer de Cuello Uterino, Comunicación.

\section{Introducción.}

La Universidad de Las Américas (UDLA) en Quito, Ecuador realizó una alianza estratégica con la Fundación CEPREME, con el fin de levantar información que permita apoyar al desarrollo de estrategias de prevención desde la comunicación y la difusión de los riesgos y tratamientos preventivos de cáncer de mama y cuello uterino en mujeres de Quito. Para ello junto a un equipo interdisciplinar de docentes, el acompañamiento de estudiantes y el apoyo técnico del personal de la Fundación se realizó una investigación misma que en este artículo presenta sus avances partiendo de los aportes de diferentes autores (Gómez et al., 2008; Ospina y Flórez, 2009; Urdaneta et al., 2013) quienes manifiestan la necesidad generar información sobre estos temas. Esto debido a que en el caso ecuatoriano el cáncer es una de las enfermedades que más vidas cobra cada año. Siendo el cáncer de mama y de cuello uterino los dos más prevalecientes en este campo. 
Tabla 1. Defunciones por cáncer en población femenina 2015

\begin{tabular}{lcc}
\hline \multicolumn{1}{c}{ LOCALIZACION } & No & \% \\
\hline Estómago & 682 & 12,89 \\
Mama & 551 & 10,41 \\
Cuello Uterino & 445 & 8,41 \\
Hígado & 376 & 7,11 \\
Colorrecto & 376 & 7,03 \\
Tráquea, Bronquios y púlmanes & 301 & 5,69 \\
Útero parte no especificada & 262 & 4,95 \\
Ovario & 221 & 4,18 \\
Páncreas & 205 & 3,87 \\
Encéfalo & 146 & 2,76 \\
Resto tumores malignos & 1.731 & 32,71 \\
TOTAL & $\mathbf{5 2 9 2}$ & $\mathbf{1 0 0}$ \\
\hline
\end{tabular}

Fuente: tomado de Ministerio de Salud Pública (2017).

Continuando con estos datos, el Ministerio de Salud Pública de Ecuador (2017) presentó la Estrategia Nacional para la Atención Integral del Cáncer en el Ecuador, donde se proponen líneas estratégicas que se recogen en la Tabla 2.

Tabla 2. Líneas de la Estrategia Nacional para la Atención Integral del Cáncer en el Ecuador

- Fomento del desarrollo de factores de protección como el autocuidado, los estilos de vida y espacios saludables.

- Fortalecimiento del control de riesgos del cáncer.

- Organizar e implementar una respuesta oportuna para el tamizaje, la detección, el diagnóstico especializado para mejorar el pronóstico y sobre vida de los pacientes con cáncer.

- Implementar intervenciones, basadas en la mejor evidencia disponible, para el tratamiento y seguimiento del cáncer en base al estadiaje de la enfermedad en los diversos niveles de atención y complejidad de los servicios de salud.

- Fortalecimiento de la rehabilitación y cuidados paliativos con calidad y calidez para los pacientes de cáncer en todos los estadios, para lograr integración interpersonal, laboral y social.

- Red especializada de atención integral oncológica para la prestación de servicios de salud en establecimientos de salud públicos y en complementariedad con los privados.

- Vigilancia Epidemiológica e Investigación para la generación de información 
estratégica nacional.

- Participación social e intersectorial.

Fuente: adaptado de Ministerio de Salud Pública (2017).

Dentro de estos espacios, surgió la necesidad de contribuir con aportes que integren reflexiones interdisciplinares con relación a estos temas y en espacial énfasis en los niveles de conocimiento y que tipo de medios preferirían para recibir este tipo de información.

\section{Método.}

\section{Unidades de medición.}

Para la realización de este estudio, se recurrió a un enfoque cuantitativo, de alcance descriptivo-exploratorio, mismo que fue recogido mediante encuestas realizadas a mujeres Estudiantes y Administrativas de la UDLA y mujeres de dos sectores ubicados en el norte y sur de Quito.

Mediante un muestreo probabilístico aleatorio simple con base en un nivel de confianza de $95 \%$ y un margen de error de 5\% del universo de mujeres en la ciudad de 1.150 .380 según datos del Instituto Nacional de Estadísticas y Censos (2010) se logró obtener un total de 384 informantes de entre 18 y 65 años.

Si bien es cierto los resultados no pretenden la generalización, contribuyen con datos de contextos mismos que permiten tener una aproximación hacia los siguientes procesos de este estudio. Cabe destacar que este avance presenta los datos parciales de la investigación que a futuro contará con otro tipo de análisis y ampliará la muestra.

\section{Instrumentos.}

El cuestionario, fue construido con el apoyo de especialistas de la Fundación CEPREME, mediante preguntas cerradas y fáciles de comprender, que permitieron la aplicación de estas por el equipo de campo.

\section{Técnicas de análisis de datos.}

Se recurrió a un análisis estadístico de alcance descriptivo-exploratorio, con el fin de interpretar los resultados mediante datos porcentuales y frecuencias expresados mediante tablas y figuras siguiendo aportes de Galvis-Lista, González-Zabala y Sánchez-Torres (2016).

\section{Procedimiento.}


La recolección de las encuestas fue asistida por miembros del equipo, mismas que se desarrollaron mediante trabajo de campo en los Sectores de Calderón al norte y Quitumbe al sur de la ciudad, además de informantes Estudiantes y Administrativas en las instalaciones de UDLA; cabe resaltar la importancia de que Instituciones de Educación Superior contribuyan al desarrollo de este tipo de investigaciones interinstitucionales que fortalecen el vínculo con la comunidad (Díaz de Rada, 2016). Posteriormente, los datos fueron ingresados a una base de datos con el fin de articular el proceso de análisis de resultados.

\section{Resultados.}

\section{Cancer de mama.}

E1 90.6\% de informantes afirman conocer qué es el cáncer de mama. Se puede apreciar que, dentro de este porcentaje, el grupo que menos conocimientos afirman tener son las mujeres de los Sectores con $29.3 \%$; seguido por Estudiantes con 33.3\% y el grupo con mayor conocimiento enuncian ser Administrativas con $37.3 \%$.

Figura 1. Informantes que afirman conocer sobre el cáncer de mama.

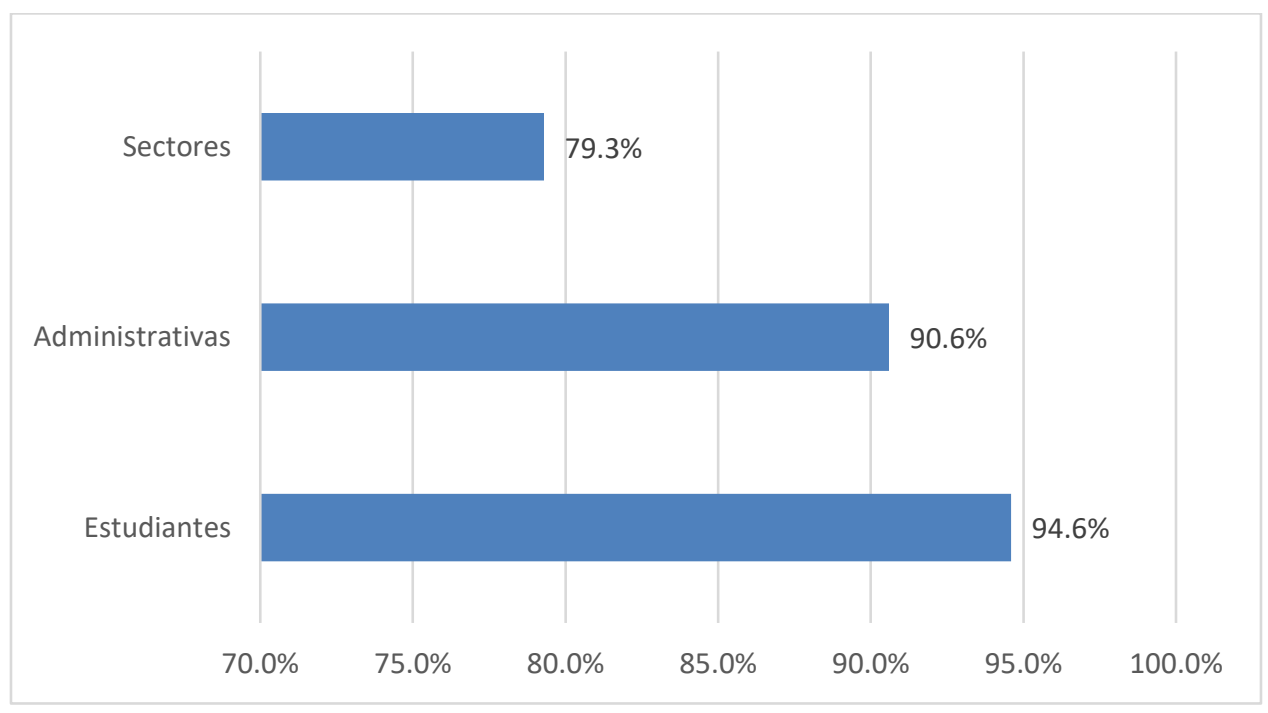

Fuente: elaboración propia. 
En todos los grupos se tiene la percepción de que los factores que más influyen en el riesgo de contraer cáncer son: los antecedentes familiares, personales y la alimentación lo cual dialoga con aportes desde diferentes países y estudios (Salas, Vega y Apodaca, 2006; Torres et al., 2008; Vivas et al., 2012). No existe una tendencia marcada en cuanto al resto de posibles respuestas. También, en todos los grupos, se considera que los factores con menor influencia son: el peso, el uso de anticonceptivos y el consumo del alcohol.

Figura 2. Factores de mayor y menor influencia en el riesgo de contraer cáncer de mama.

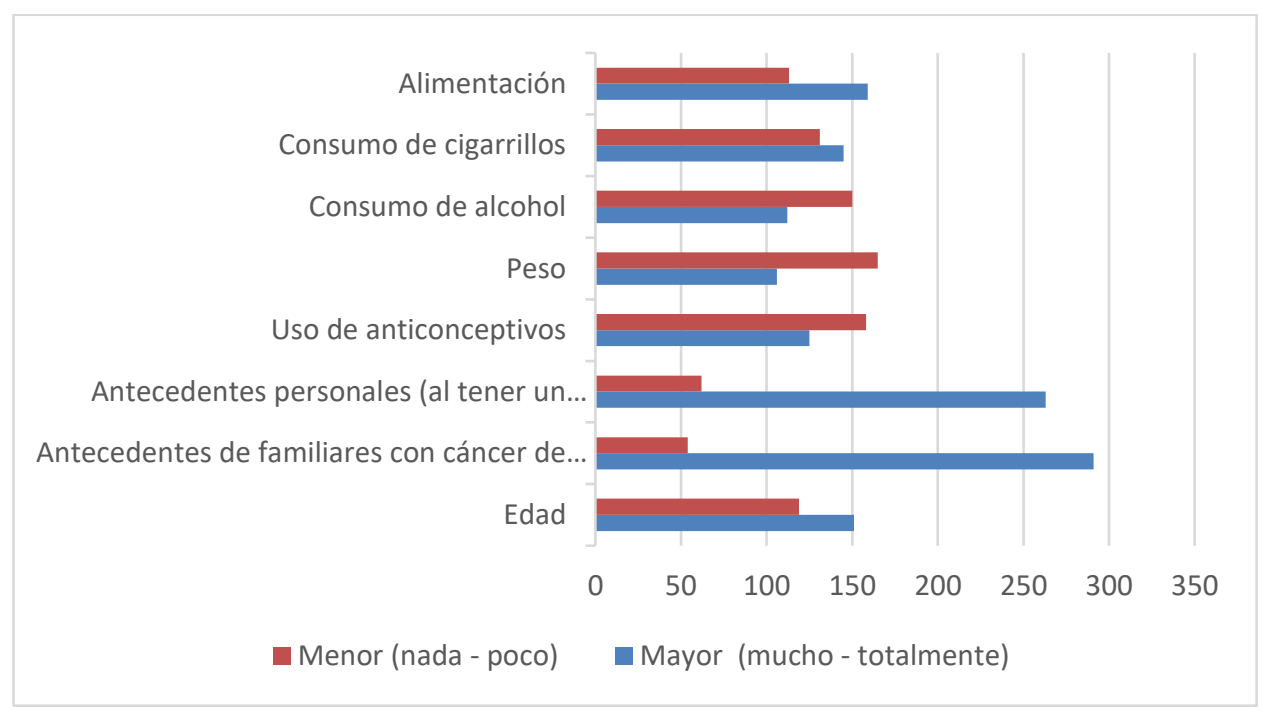

Fuente: elaboración propia.

Dentro de quienes afirmaron conocer qué es el cáncer de mama, el $89 \%$ menciona al autoexamen de senos como método de prevención. De este porcentaje las mujeres de los Sectores son quienes menos identifican este método de prevención con $29 \%$, seguidas por Estudiantes con $35.8 \%$ y Administrativas con 39.6\%. En relación a ello Shur (2006) menciona la relevancia de tomar conciencia sobre el autoexamen como estrategia de prevención. De quienes afirmaron conocer este método, el $68.6 \%$ menciona que conoce la manera correcta de realizarse un autoexamen. De los grupos de informantes se pueden observar frecuencias y porcentajes de respuestas por grupos en la Tabla 3. 
Tabla 3. Informantes que conocen como realizar el autoexamen de senos.

\begin{tabular}{lcccc}
\hline & Administrativas & Estudiantes & Sectores & Total \\
\hline Frecuencia & 106 & 72 & 59 & 237 \\
Porcentaje & $44.3 \%$ & $30.9 \%$ & $24.8 \%$ & 100 \\
\hline
\end{tabular}

Fuente: elaboración propia.

Como parte de las técnicas de prevención, se consultó cuál consideraban era la frecuencia adecuada para realizar el autoexamen de seno. De quienes respondieron que conocen cómo realizarlo la mayor cantidad de mujeres en todos los grupos percibe que el autoexamen se debe realizar cada mes, el segundo grupo de mayor porcentaje considera que el examen debe ser cada semestre y el tercero enuncia cada quince días. Los porcentajes pueden observarse en la Tabla 4 . De igual manera el 59\% de informantes consideran que las edades para empezar a realizarse el autoexamen son entre los 19 y 25 años.

Tabla 4. Frecuencia en que deben realizar autoexamen de seno.

\begin{tabular}{lc}
\hline \multicolumn{1}{c}{ Frecuencia } & Porcentaje \\
\hline Una vez al mes & $47,4 \%$ \\
Una vez cada 15 días & $18,1 \%$ \\
Una vez cada dos meses & $11,8 \%$ \\
Una vez cada seis meses & $18,5 \%$ \\
Otro & $4,2 \%$ \\
\hline
\end{tabular}

Fuente: elaboración propia.

Se buscó conocer, si dentro de la periodicidad en la que se debe realizar el autoexamen, existe algún momento del ciclo menstrual que la mujer considera que es mejor hacerse el autoexamen, y se obtuvo que la mayor parte de mujeres en todos los grupos encuestados consideran que cualquier día del ciclo es apropiado con $38 \%$, seguido por tres días después de la menstruación con 30\%; ello dialoga con resultados de estudios realizados en Colombia por Manrique (2012).

A la consulta sobre el nivel de conocimiento de distintas formas de prevención del cáncer de mama; se pudo identificar, que los tres primeros enunciados son: chequeos ginecológicos constantes, realización de mamografías y estilo de vida saludable. Dentro de las opciones que menor frecuencia obtuvieron se encuentran: vacunas, realización responsable del autoexamen mamario y alimentación saludable. 
Figura 3. Métodos de prevención para el cáncer de mama.

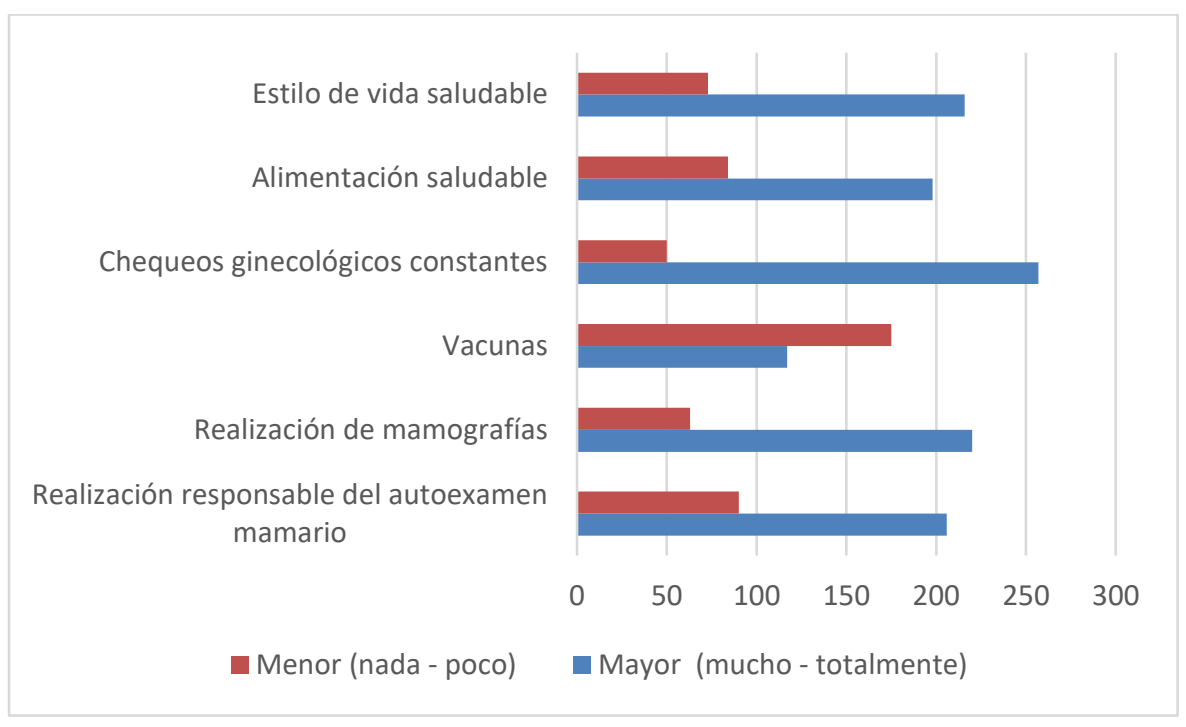

Fuente: elaboración propia.

Dentro de las encuestadas, el 95\% de informantes han recurrido donde un especialista para realizarse un control de mamas, de ellas las Administrativas son quienes más han acudido con $42 \%$, seguidas por Estudiantes con $31 \%$ y mujeres de Sectores con $27 \%$.

De las mujeres que mencionan tener algún tipo de información sobre cáncer de mama, las tres principales fuentes han sido: Profesional Médico en un 45\%, siendo la segunda fuente de información los Familiares y Amigos con 24.7\% y TV, radio y prensa con 15\%.

Figura 4. Fuentes de información sobre cáncer de mama.

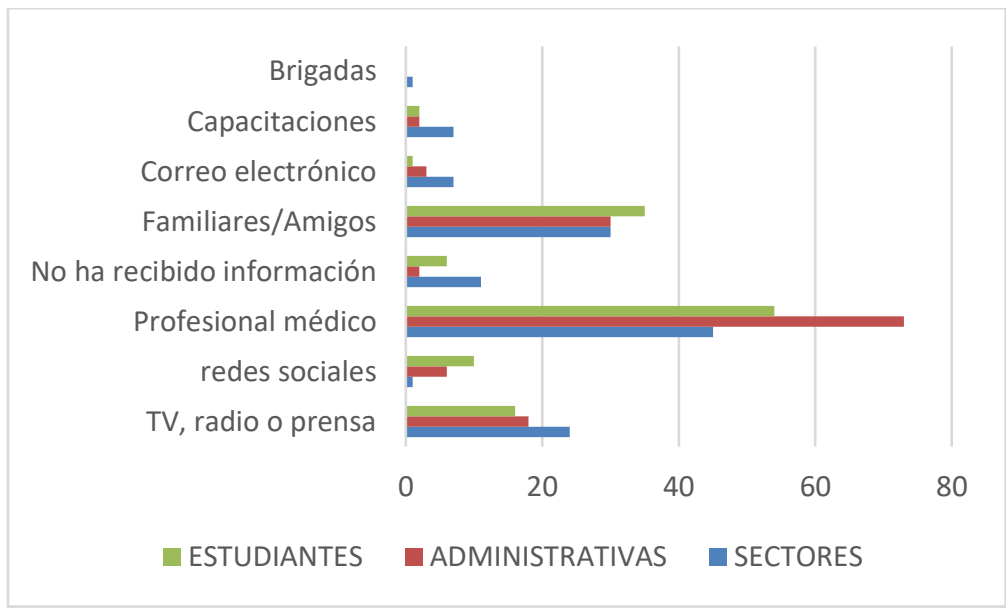

Fuente: elaboración propia. 
Se pudo identificar también que todos los grupos preferirían conocer sobre estos temas principalmente mediante capacitaciones; brigadas, redes sociales y Tv, radio y prensa, lo cual dialoga con otros aportes (Manrique, 2012; Sánchez, 2014; Apolo et. A1., 2014), donde se incentiva reforzar estrategias de educación y motivación a partir de lugares cercanos como vivienda, trabajo y redes sociales.

Figura 5. Medios de preferencia para recibir información.

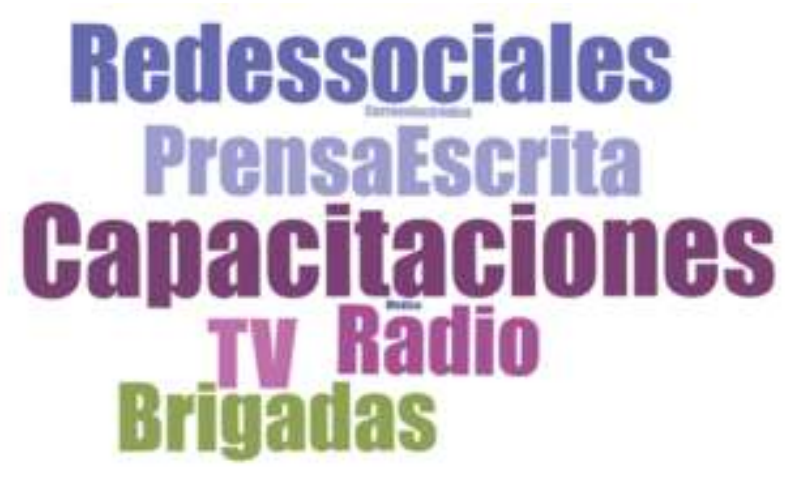

Fuente: elaboración propia.

\section{Cáncer de cuello uterino.}

De las informantes en todos los grupos el $79.8 \%$ afirman conocer sobre el cáncer de cuello uterino; se puede observar en la Figura 6 las respuestas por grupos, donde las Administrativas mencionan tener más conocimientos seguidas por Estudiantes y Sectores.

Figura 6. Porcentaje de mujeres que afirman tener información sobre el cáncer de cuello uterino.

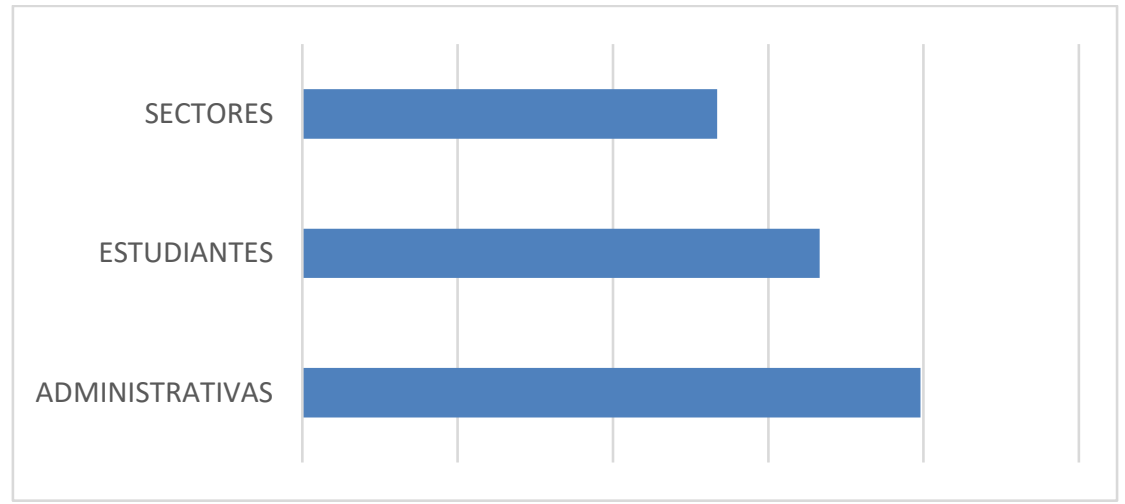

Fuente: elaboración propia. 
E1 93\% de informantes menciona conocer qué es el Virus del Papiloma Humano VPH, pese a ello el $15 \%$ de quienes afirmaron tener este conocimiento desconocen que el Cáncer de Cuello Uterino es causado por este Virus. Esta última respuesta indica que el conocimiento sobre este tipo de cáncer es amplio, pero que su relación con el Papiloma no necesariamente está claro entre las mujeres encuestadas, siendo un dato que dialoga con estudios realizados en Venezuela por Sánchez (2014).

En todos los grupos, se tiene la percepción de que los factores que más influyen en el riesgo de contraer cáncer de cuello uterino son: los antecedentes familiares, personales y consumo de alcohol. De los factores con menor selección constan: uso de anticonceptivos, consumo de cigarrillos y alimentación.

Figura 7. Factores de mayor y menor influencia en el riesgo de contraer cáncer de cuello uterino.

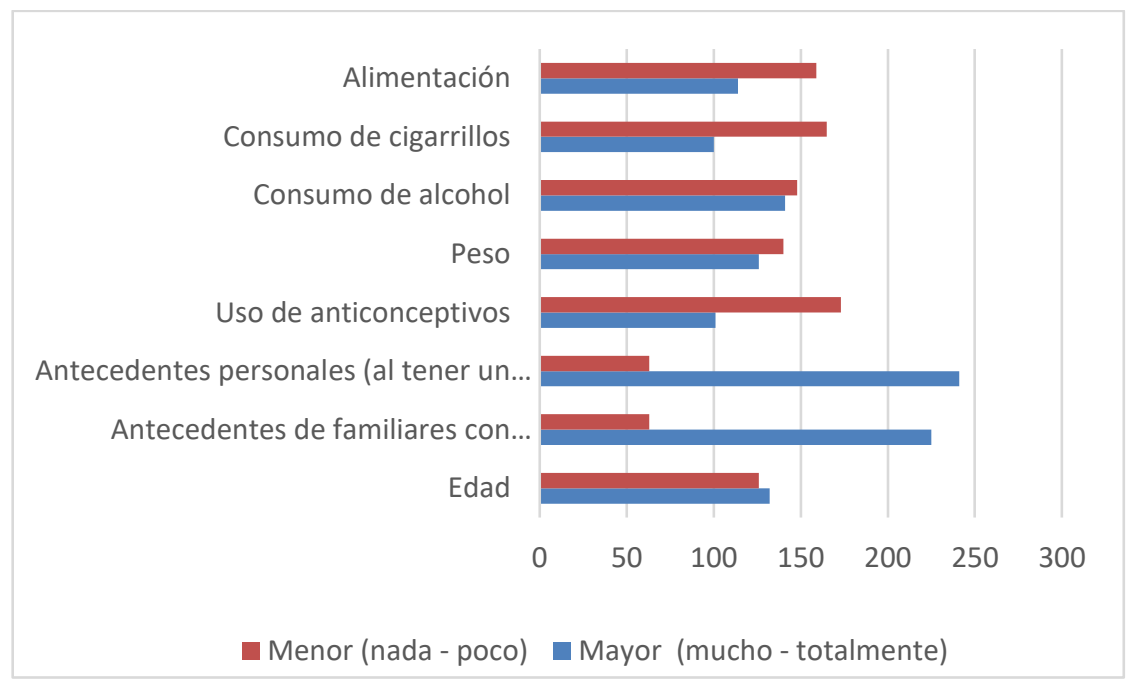

Fuente: elaboración propia.

Continuando con el análisis, se puede mencionar que el $89 \%$ de las informantes conocen de qué se trata el examen de Papanicolaou. En cuanto a los conocimientos sobre formas de prevención del cáncer de cuello uterino, se puede observar que con mayor frecuencia se encuentran: Papanicolaou, chequeos ginecológicos constantes y una alimentación saludable. De los mencionados en menor medida son: estilo de vida saludable, uso del preservativo y vacunas (Marañón, 2017). 
Figura 8. Métodos de prevención para el cáncer de cuello uterino.

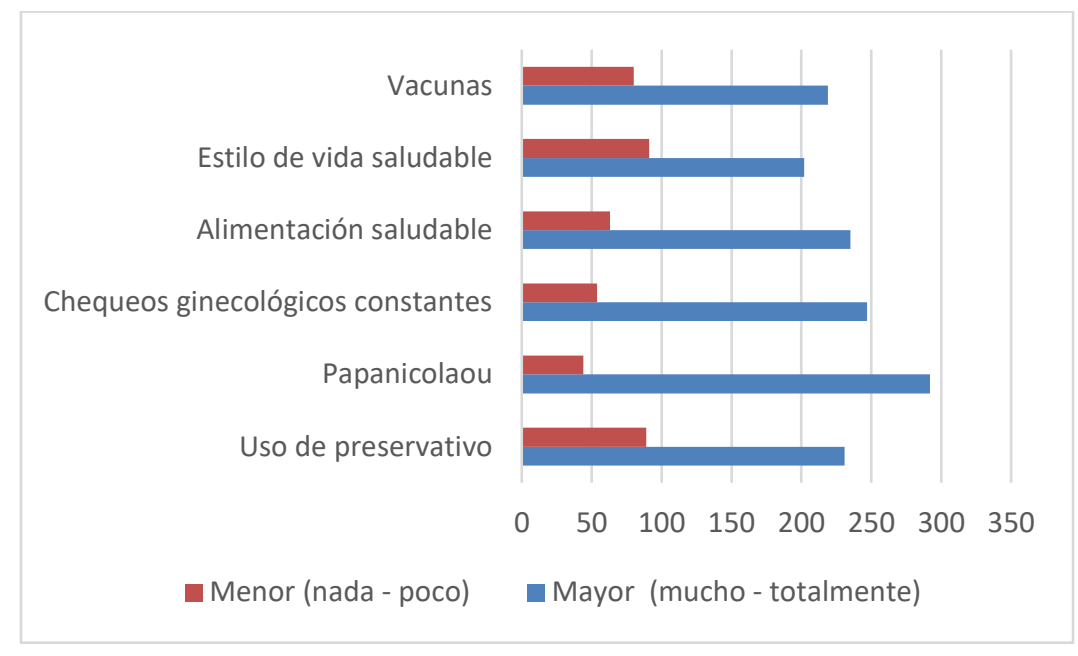

Fuente: elaboración propia.

Adicionalmente, se obtuvo que el grupo de mujeres de Sectores es el grupo que menos se ha realizado el examen de Papanicolaou con un $27.7 \%$, seguidas por Estudiantes 33\%, mencionar haberse realizado con mayor frecuencia el examen son Administrativas con $39 \%$. De las mujeres que se han realizado el examen el 57,8\% menciona que se debe realizar entre cada seis meses y un año.

Para los tres grupos encuestados, las fuentes de información más comunes son: Profesional médico, Familiares/Amigos y TV, radio y prensa. De estos grupos la mayoría desea ser informado bajo los mismos canales que se mencionaron para el cáncer de mama: Capacitaciones y Brigadas y por tanto se siguen las propuestas de Urdaneta et al. (2013), para identificar la relevancia que enuncian diferentes estudios con miras hacia la prevención y control de cáncer de cuello uterino.

\section{Conclusiones.}

- A partir de los abordajes presentados, se puede concluir que en general, los tres grupos de encuestadas (Estudiantes, Administrativas y Sectores) presentan características similares con relación a los temas analizados sin mostrar marcadas diferencias generacionales ni geográficas.

- En cuanto al nivel de conocimiento general se observa que aquellas personas que afirmaron desconocer qué es el cáncer de mama, también afirmaron desconocer qué es el cáncer de cuello uterino, virus de Papiloma Humano y Papanicolaou; por tanto, se puede establecer que los niveles de conocimientos no son suficientes tomando en 
cuenta que estos son dos de los factores de mayor mortalidad de mujeres en Ecuador.

- Las informantes que no se realizaron un control de mamas por un profesional tienden a tampoco haberse realizado el autoexamen de senos ni la prueba del Papanicolaou.

- Se observa que las Administrativas son el grupo que realiza más prácticas de prevención de cáncer de mama, mientras que las Estudiantes tienden con mayor frecuencia a no realizarse la prueba de Papanicolaou. No existe asociación en cuanto a las prácticas de prevención de las personas encuestadas en los Sectores.

- Surgen importantes datos sobre la relevancia que toma la comunicación en temas de salud, y la necesidad de construir planes que giren alrededor de estrategias de contacto directo como Capacitaciones o Brigadas en lugares de trabajo o Sectores que sean pertinentes, articuladas e integrales desde la educación, información y motivación con el fin de fortalecer prácticas de prevención en la comunidad. Vale recalcar que estos datos dialogan con las propuestas de la Estrategia Nacional para la Atención Integral del Cáncer (Ministerio de Salud Pública, 2017).

- Se destaca como punto trascendental el papel que toman las Instituciones de Educación Superior y su vínculo con la comunidad, debido a que desde un trabajo transdisciplinar académico se puede contribuir a la reflexión y a realizar estudios que sean un aporte a las políticas de salud.

\section{Referencias bibliográficas.}

Apolo. D., Murillo, H. \& García, G. (2014). Comunicación 360: herramientas para la gestión de imagen e identidad. Quito: Universidad Tecnología Equinoccial.

Díaz de Rada, V. (2011). Encuestas con encuestador y autoadministradas por internet ¿Proporcionan resultados comparables? Reis, (136), 49-90.

Galvis-Lista, E., González-Zabala, M., \& Sánchez-Torres, J. (2016). Un estudio exploratorio sobre el estudio de implementación de procesos de gestión del conocimiento en organizaciones desarrolladoras de software en Colombia. Revista EAN, (80), 73-90.

Gómez, J., Altagracia, M., Kravzov, J., Cárdenas, R., \& Poo, C. (2008). Cáncer de mama y las actuales alternativas de tratamiento. Revista Mexicana de Ciencias Farmacéuticas, 39(3), 58-70.

Instituto Nacional de Estadísticas y Censos. (2010). Población y Demografía. Disponible en: http://www.ecuadorencifras.gob.ec/censo-de-poblacion-y-vivienda/ 
Manrique, F., Ospina, J., Vega, N., Morales, A., \& Herrera, G. (2012). Factores asociados a la práctica correcta del autoexamen de mama en mujeres de Tunja (Colombia). Investigación y educación en enfermería, 30(1), 18-27.

Marañón, T., Mastrapa, K., Flores, Y., Vaillant, L., \& Landazuri, S. (2017). Prevención y control del cáncer de cuello uterino. Correo Científico Médico, 21(1), 187-203.

Ministerio de Salud Pública. (2017). Estrategia Nacional Para La Atención Integral Del Cáncer En El Ecuador. Disponible https://aplicaciones.msp.gob.ec/salud/archivosdigitales/documentosDirecciones/dnn/archiv os/ac_0059_2017.pdf, 2017.

Ospina, D., y Flórez Marín, N. (2009). Producción científica sobre cáncer cérvico-uterino y de mama en Colombia, 1994-2004. Estudio según la Base de Productos Nacionales XACTA de Colciencias, Latreia, 22(2), 112-121.

Sánchez, N. (2014). Las redes sociales en Internet y su impacto en la Salud Pública. Revista Cubana de Información en Ciencias de la Salud, 25(2), 143-144.

Shur, J. (2006). Conocimientos, actitudes y prácticas sobre auto-examen de mama y mamografía como detección precoz del cáncer de mama en mujeres leonesas (Doctoral dissertation). León: Universidad Nacional Autónoma de Nicaragua.

Salas, I., Vega, B., \& Apodaca, E. (2006). Factores de riesgo para la presentación de cáncer de mama en el Centro Médico Nacional Siglo XXI Chihuahua, México. CIMEL Ciencia e Investigación Médica Estudiantil Latinoamericana, 11(2), 62-66.

Torres, L., Sarmiento, J., Sánchez, A., Pacheco, O., Capín, N., \& Rojas, P. (2008). Cáncer de mama. Caracterización durante el decenio 1996-2005. Medisur, 5(3), 36-42.

Urdaneta, J., Nava, M., García, J., Cepeda, M., Zambrano, N., Salazar, J., \& Mujica, A. (2013) Conocimiento del Cáncer de Cuello Uterino y Hallazgos Citológicos en Mujeres de Estratos Socioeconómicos Bajos. Revista Venezolana de Oncología, 25(4), 211-228.

Vivas, V., Torres, N., Esguerra, L, Torres, C., Mojica, I., Mendoza, K. \& Rayón, M. (2012). Conocimientos, actitudes y prácticas de prevención del cáncer de mama. Revista Ciencia y Cuidado, 9(2), 43-51.

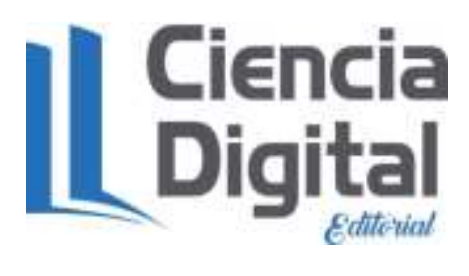


Para citar el artículo indexado.

Hidalgo P., Toledo E. \& García J. . (2018). conocimientos sobre cáncer de mama y cáncer de cuello uterino en mujeres de quito: avances de investigación. Revista electrónica Ciencia Digital 2(3), 117-129. Recuperado desde: http://cienciadigital.org/revistacienciadigital2/index.php/CienciaDigital/article/view/141/12 $\underline{6}$

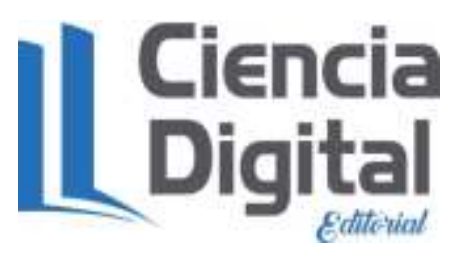

El artículo que se publica es de exclusiva responsabilidad de los autores y no necesariamente reflejan el pensamiento de la Revista Ciencia Digital.

El articulo queda en propiedad de la revista y, por tanto, su publicación parcial y/o total en otro medio tiene que ser autorizado por el director de la Revista Ciencia Digital.
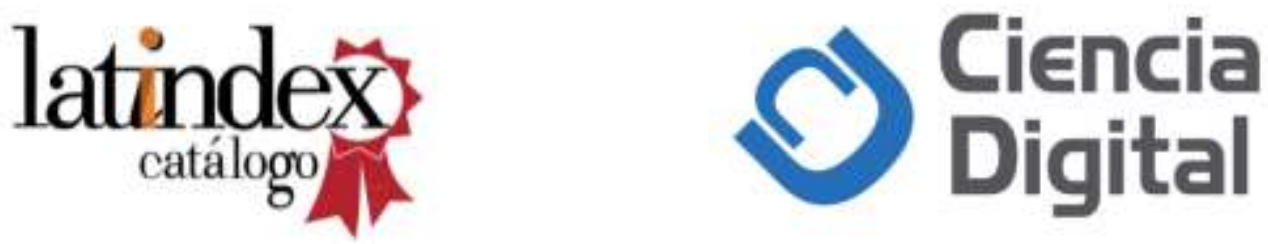